\section{Research Square}

\title{
Erenumab during pregnancy: a case report in a patient with chronic migraine
}

\section{Luisa Fofi}

Istituto di Ricovero e Cura a Carattere Scientifico San Raffaele Pisana

\section{Gabriella Egeo}

Istituto di Ricovero e Cura a Carattere Scientifico San Raffaele Pisana

\section{Cinzia Aurilia}

Istituto di Ricovero e Cura a Carattere Scientifico San Raffaele Pisana

Piero Barbanti ( $\sim$ piero.barbanti@sanraffaele.it )

IRCCS San Raffaele Pisana https://orcid.org/0000-0002-5670-3755

\section{Case report}

Keywords: Erenumab, pregnancy, safety, newborn, case report

Posted Date: August 3rd, 2020

DOI: https://doi.org/10.21203/rs.3.rs-50684/v1

License: (c) (1) This work is licensed under a Creative Commons Attribution 4.0 International License. Read Full License 


\section{Abstract}

Background: Migraine is very common in women of childbearing age. Erenumab is a first-in-class antiCGRP monoclonal antibody with a a favorable efficacy/tolerability profile. However, there are very limited data on its effects during pregnancy.

Case presentation: We report a case of a 37-years-old woman affected by chronic migraine who became pregnant during erenumab treatment. At the time of her first visit at our Unit she was on prophylaxis with sodium valproate $600 \mathrm{mg} /$ day reporting low efficacy (18 headache days/month) and tolerability (weight gain). She had already failed 5 prior preventative treatments. On the same day started erenumab $70 \mathrm{mg}$, tapering down valproate. Erenumab was rapidly effective, reducing migraine frequency (7 days/month) since the first administration. On the follow-up visit scheduled for the fifth erenumab administration, the patient informed us of her pregnancy, documented by appropriate serological test the day before. Erenumab was immediately stopped. The last erenumab administration was made on 04/07/2019, probably corresponding to the first two weeks of pregnancy. Serological fetal DNA test was normal. During the I pregnancy trimester the frequency of migraine increased up to 10 days/month, markedly improving and progressively disappearing during the II-III trimesters. Migraine attacks started again from the 34th week of pregnancy throughout delivery and breast feeding. No other adverse event or complication occurred during pregnancy. The baby, born by vaginal birth in healthy conditions, developed breastfeeding jaundice which progressively normalized over time.

Conclusions: We document no complications, abnormalities, or adverse events in both the mother and the child, and a physiological normal course of pregnancy, delivery and breastfeeding in the first report of erenumab exposure during pregnancy in a migraine patient. More data from clinical studies and postmarketing surveillance are necessary to confirm our findings.

\section{Background}

Migraine is very common in women of childbearing age, reaching a prevalence of $25 \%{ }^{1}$. The preventive treatment of migraine in women of reproductive age must be carefully chosen, since some oral drugs like valproate and topiramate have known teratogenic effects.

New anti-Calcitonin Gene-Related Peptide (CGRP) monoclonal antibodies represent a new era in the preventive treatment of migraine, because of their favorable efficacy and tolerability profile. However, there are very limited data on their effects during pregnancy, therefore - since CGRP is a potent vasodilator potentially involved in the blood flow regulation in the uteroplacental system - theoretical concerns exist ${ }^{2}$.

Erenumab - a fully human monoclonal antibody targeting the receptor of the CGRP and the first drug in this new class - showed to be effective and well tolerated in episodic and chronic migraine ${ }^{3-4}$. In a preand post-natal development study in cynomolgus monkey, erenumab administration from gestation day 20-22 through birth induced no adverse effects on the pregnancy and the embryo-fetal growth and 
development at exposures 17 -fold the human dose of $140 \mathrm{mg} / \mathrm{month}^{5}$. Nevertheless, due to the lack of clinical data and the theoretical role of CGRP in regulating uterine vascular resistance, the use of erenumab during pregnancy is currently not recommended.

This is the first report on the occurrence of pregnancy during erenumab treatment in a patient affected by chronic migraine.

\section{Case Presentation}

This is a 37-year-old woman, married, working as a lawyer. Her medical history was positive for endometriosis, polycystic ovarian and an untreated anxiety disorder. She had no previous pregnancy or abortions. The patient was affected by migraine without aura since the age of 6 . Until early adolescence, migraine had a frequency of 4 days per month, and was responsive to non-steroidal anti-inflammatory drugs. Migraine started worsening at the age of 16, becoming chronic (15-20 days/month) without medication overuse. The putative chronification factors were stress and anxiety. The attacks were successfully treated with triptans. Over the course of the disease, the patient used amitriptyline, cinnarizine, flunarizine, propranolol, metoprolol and topiramate, but all these treatments proved ineffective, or had unbearable side effects, which led to a premature discontinuation. At the time of her first visit to our Unit, she was on prophylaxis with sodium valproate $600 \mathrm{mg} /$ day, showing a migraine frequency of 18 days per month. General and neurological examination, brain MRI scan and blood tests were unremarkable. On 4/4/2019 the patient discontinued valproate due to its low efficacy and to weight gain, and started erenumab $70 \mathrm{mg}$ subcutaneously every 28 days. Subsequently she was checked at our Unit on a monthly basis. Erenumab was rapidly effective, reducing the frequency of migraine to 7 days per month since its first administration. On 8/8/2019, at the follow-up visit scheduled for the fifth erenumab administration, the patient informed us of her pregnancy, documented on $7 / 8 / 2019$ by positive deep stick urine test and high beta-HGC plasma level $(32257 \mathrm{mUl} / \mathrm{mL})$. The erenumab treatment was immediately stopped. The last erenumab administration was performed on $4 / 7 / 2019$, probably in correspondence with the first two weeks of pregnancy, since her last period occurred from 8 to 13 June.

The patient underwent all routine gynecological investigations, and spontaneously decided against amniocentesis. The serological fetal DNA test was normal.

During the first trimester of pregnancy, the frequency of migraine increased up to 10 days/month. During the second trimester, migraine showed a marked improvement, and during the fifth, sixth and seventh months she reported no attacks. Migraine attacks started again with a frequency of 4-5 days/month from the 34th week of pregnancy, throughout delivery and breast feeding. No blood pressure alteration, nor any other adverse event or complication, occurred during pregnancy. The baby - a male - was born by vaginal birth on $20 / 3 / 2020$ (38th week +5 days) in healthy conditions and with no malformation or congenital anomalies. His weight was $3,300 \mathrm{gr}$, his length $51 \mathrm{~cm}$, his head circumference $35 \mathrm{~cm}$; the Apgar score was 9 at birth and 10 after 5 minutes. An exclusive breast-feeding regimen was adopted. During the first days, the newborn developed breastfeeding jaundice (bilirubin $18.9 \mathrm{mg} / \mathrm{dL}$ ), which 
progressively normalized over time. At the age of 40 days, the newborn measurements were normal, showing a weight of $5,600 \mathrm{gr}$, a length of $56 \mathrm{~cm}$ and a cranial circumference of $39 \mathrm{~cm}$.

\section{Discussion}

To the best of our knowledge, this is the first description of erenumab exposure during the first two weeks of pregnancy in a migraine patient. We documented no complications, abnormalities, or adverse events in both the mother and the child, and a physiological normal course of pregnancy, delivery and breastfeeding. Before planning her pregnancy, a woman should stop erenumab early, due to its long halflife (28 days) ${ }^{6}$. There is no adequate data on the developmental risk associated with the use of erenumab in pregnant women; more data from clinical studies and post-marketing surveillance are necessary to confirm our findings.

Written informed consent was obtained from the patient for the publication of this case report.

\section{Abbreviations}

CGRP: calcitonin gene-related peptide.

\section{Declarations}

\section{Aknowledgment}

The authors would like to thank T\&T S.a.s for native English check and Springer Healthcare Italia S.r.l. for publication fees assistance. These supports were funded by Novartis Farma.

\section{Key findings}

- This is the first report of accidental erenumab exposure during the first two weeks of pregnancy in a patient affected by chronic migraine.

- No complications, abnormalities, or adverse events in both the mother and the child, and a physiological normal course of pregnancy, delivery and breastfeeding were documented

- Before planning her pregnancy, a woman should stop erenumab early, due to its long half-life

- More data from clinical studies and post-marketing surveillance are necessary to confirm our findings.

\section{Conflict of Interest:}

Piero Barbanti received travel grants, honoraria for advisory boards, speaker panels or clinical investigation studies from Alder, Allergan, Bayer, ElectroCore, Eli-Lilly, GSK, Lusofarmaco, MSD, Novartis, Stx-Med, Teva, Visufarma. 
Luisa Fofı received travel grants and honoraria for advisory boards from Teva, Eli-Lilly and Novartis

Cinzia Aurilia received travel grants from Eli-Lilly, FB-Health, Lusofarmaco and Teva;

Gabriella Egeo received travel grants and honoraria from Eli-Lilly, Novartis, New Penta and Ecupharma;

\section{References}

1. Amundsen $\mathrm{S}$, Nordeng $\mathrm{H}$, Nezvalova-Henriksen $\mathrm{K}$, et al. Pharmacological treatment of migraine during pregnancy and breastfeeding. Nat Rev Neurol 2015; 11(4):209-19

2. European Medicines Agency: EMA/CHMP/413393/2018 - Aimovig CHMP Assessment Report, https://www.ema.europa.eu/en/documents/assessment-report/aimovig-epar-public-assessmentreport_en.pdf, May 2018.

3. Goadsby PJ, Reuter U, Hallström Y, et al. A con- trolled trial of erenumab for episodic migraine. N Engl J Med. 2017;377:2123-2132.

4. Tepper $S$, Ashina $M$, Reuter $U$, et al. Safety and efficacy of erenumab for preventive treatment of chronic migraine: A randomised, double-blind, placebo-controlled phase 2 trial. Lancet Neurol. 2017; 16:425-434.

5. Bussiere JL, Davies R, Dean C, Xu C, Kim KH, Vargas HM, Chellman GJ, Balasubramanian G, RubioBeltran E, MaassenVanDenBrink A, Monticello TM. Nonclinical safety evaluation of erenumab, a CGRP receptor inhibitor for the prevention of migraine. Regul Toxicol Pharmacol. 2019 Aug;106:224238. doi: 10.1016/j.yrtph.2019.05.013. Epub 2019 May 11

6. https://www.accessdata.fda.gov/drugsatfda_docs/label/2018/761077s000lbl.pdf 